\title{
Understanding the use of paper and online logbooks for final year undergraduate engineering projects.
}

\author{
Manish Malik, \\ School of Engineering, \\ University of Portsmouth, \\ Portsmouth, U.K. \\ Manish.malik@port.ac.uk
}

\begin{abstract}
In industry an engineer is often required to keep a logbook for recording developments within projects. In higher education, logbooks are a commonly used tool thought to be one that encourages active independent learning and reflective thinking. In School of Engineering, at University of Portsmouth, paper and more recently online logbooks have been in use for recording work for final year projects and project based learning tasks. The work presented here benefits from a unique opportunity within the School of Engineering, where online logbooks alongside traditional paper based logbooks are being used within final year projects. A recent cohort of students $(\mathrm{N}=127)$ on ENG600 project module was given the option, through their Supervisors, to use paper logbooks and or online logbooks for recording their work. This work aims to investigate the use of both paper and online logbooks. A mix of Qualitative Research methods and quantitative techniques will be used in this project. The use of content analysis will provide an insight into student reflections and their motivations for using their logbook. Furthermore focus groups, involving live editing of documents in an individual and collaborative fashion, will be used to gather more data for analysis. Quantitative methods (questionnaire, analytics and quantitative content analysis) will also be used in this study. When this work is completed, it will provide guidance and comparison on using the two types of logbooks, backed by knowledge of student motivations and approaches.
\end{abstract}

Keywords - Capstone projects, Final year projects, Online logbook, Enginee's notebook, Project supervision.

\section{INTRODUCTION}

Some of the qualities of a good engineer include being a lifelong and independent learner, a problem solver and being self-motivated and self-critical [1]. Engineers, when working on different projects for customers, are often required to use logbooks to record development within a project [2, 3, and 4]. These logbooks are useful both during and after the project. The engineer or a team may use a logbook to develop and record their ideas and improve their designs during the work [2]. Later, in case of a customer complaint or product problems the logbook offers an insight into the engineers thinking and a way to identify problems [2].

Final year undergraduate projects present students with the opportunity to develop and practice the skills needed to be a good engineer by being self-motivated and self-critical and practicing problem solving skills. In the School of
Engineering, final year project logbooks (paper based ones) have been in use since late 1990s. Students use these to record plans, different considerations they make, actual work done, reflections on the work done and any alterations to their plans. The process of reflections and considering why to take a certain path and not other alternatives, promote independent thinking in students. The logbook, alongside other evidence, is submitted for assessment purposes.

Logbooks are widely used within higher education settings in engineering, medical and teacher education fields. A simple search limited to ".ac.uk" websites for the term "logbook" results in several relevant results detailing what a logbook is, why and where it is used and so on [1, 3, 5 and 10]. The same search can also be performed to sample practice in other institutions outside of the UK. A search of this nature with relevant URL ending (for example ".edu" or ".edu.au") and alterations on the term "logbook" such as "notebook", "diary", "laboratory notebook" etc. resulted again in several relevant pages from US, Canadian, European, and Australian universities. Several US, and UK based engineering schools have posted guidance on the use of logbooks within projects and or project based learning modules on their school websites. Despite several institutions currently promoting and using logbooks, there seems little evidence within engineering education research literature that reports evaluation studies on the use of paper and or online logbooks. Literature review from medical education field highlights different studies done on feasibility of logbook systems and other areas but none looking at the motivations of students [7].

Usually there are marks associated with the logbooks that may motivate and encourage some students to actively keep logbooks up to date. A good logbook will describe both the work and the reasons behind doing it. It will also have reflections and records of critical incidents that evidence development of the work and the student.

However, the student's approach to record keeping varies. For some, keeping a logbook up to date may be seen as unnecessary laborious work and some may detest this work for other reasons. Equally there are students who meticulously record every single detail and record their decision making within their logbooks. Besides, there are issues such as safekeeping/loss of logbooks and having access to one's logbook 
when needed. Ideas not recorded may be forgotten and disadvantage a students' overall work as it is time constrained.

This project aims to add to the existing Engineering Education literature and overall literature on the use of logbooks (paper and online) and student motivation in using these and the way they use the logbooks.

The School of Engineering is in a unique situation where students were given a choice, in agreement with their Supervisors, to use either a combination of online and paper logbooks or just online logbooks. They were asked to discuss this with their Supervisor and decide if they wanted them to use paper logbooks for their work. This resulted in a control group as three Supervisors told all their students to use paper logbooks and the students all did so. These students were required to record their meeting minutes in the online logbooks and all other work in their paper logbooks.

The paper logbook takes the form of a hardbound notebook given to students from the school store at the start of their project. The school is using Google Docs for online logbooks for this project. These are created automatically for the students also at the start of the project and again at regular intervals as previous logbooks are locked out. Some colleagues in the school have been using Google docs and other wiki platforms for this purpose since around 2007. It is only now that the practice of using online logbooks for the entire ENG600 cohort (apart from the control group) has been tried out.

The author and other colleagues in the School of Engineering have already published work on the sole use of online logbook to enhance project supervision and guidance [5]. This paper will not investigate the benefits of using the two types of logbooks for supervision and guidance. Readers should read [5] if benefits of logbooks for supervision are of interest to them.

This paper presents the research design and the rationale for the current focus of the study. It also presents some preliminary data taken from the available online logbooks that highlight some trends, which will aid in answering the research questions in this research. This work when completed should be of interest to engineering academics who supervise projects and to the project coordinators. Several schools across UK and US seem to be using logbooks but the underpinning research on the efficacy of these within engineering education is virtually nonexistent. This work should also be of interest to academics from medical and other science disciplines where logbooks are widely used in education.

\section{RESEARCH DESIGN \& METHODOLOGY}

\section{A. Research Questions}

Use of logbooks has already been studied within engineering organizations [3]. Logbooks are in use by students in medical and engineering courses [5, 6, and 7]. Past studies have focused on benefits of logbooks for supervision and on student's overall achievement in projects. This work aims to investigate the use of logbooks in general and student motivation in their use of paper and/or online logbooks. Below are the two research questions that this work focuses on:
1) What are the intrinsic and extrinsic motivations for a final year undergraduate engineering student's use of paper or online logbooks for their project work?

2) How do final year undergraduate engineering students use their paper or online logbooks for within their project work?

\section{B. Design, Methodology and Rationale}

As governed by the research questions above, the aims here are to,

1) Investigate students' motivation in the use and upkeep of the two types of logbooks.

2) Investigate what, how and when students update their logbooks and highlight the differences in the use of the two types of logbooks.

The logbooks itself will be used in this project for analysis and studying the student motivation. However, student motivation is a function of both intrensic and extrinsic factors. This calls for the use of mixed methods for this research. Where unique individual intrensic factors affecting a student's motivation are best captured through an interpretive apporach and the extrensic factors are best captured through a positivist approach.

In order to capture some of the qualitative data focus groups will be used. Each participant in the focus group will be given a Google Document with a set of open ended questions to complete. These answers will be collected during and or prior to the online focus group event. Later, to aid discussion the key points from each participant will be selected by the researcher and displayed anonymously through a shared document that will allow students to comment on these points verbally and by typing in an online conference. The data will be annonymised and presented, safeguarding identity of the participants. The disucssion that follows will be recorded and transcribed for analysis.

Quantitative data will also be collected for analysis to study the extrinsic factors effecting student motivation and use of the logbooks. Students will be asked how often (Daily, Weekly, or Monthly etc) they recorded within their logbooks, did they do so in real time or retrospectively or both and why. Google forms will be used to create a Likert Scale Questionnaire to collect this data. Arrangements will be in place to ensure one student gets to input only one response. Additionally, Google Docs revision history feature will be used to compare with questionaire data and gain insight on the frequency of a student's actual use of their online logbooks. This will also show if the student is completeing their logbook real time or retrospectively. Of course this cant be done with paper logbooks so the comparison with questionnair data will only be possible for online logbooks.

Furthermore, coding and analysing the occurances of codes within the doucments will be carried out. This should help identify what students are recording, for example reflections, 
skecthes, calculations, research, plan, copying and pasting text or images etc. This will help compare the two logbook types and highlight any evidence that suggest certain types of entries are only possible in each type of logbook. Actual student report content will be compared with what they recorded in their logbooks. This will help understand how much of the work recorded in the logbooks is used by the students in their final submitted work.

All this will throw more light on the student's intrinsic and extrinsic motivations. For example, a student who benefits from keeping a log of their work may record in real time or very regualrly and througout the project period. The words used within the logbook may indicate that the student has benefitted from this process and a regular use of reflective language could also point in this direction.

Informed consent has already been acquired from more than 48 randomly chosen participants $(37.7 \%, \mathrm{~N}=127)$. Due to the nature of data being a mix of qulitative and quantitative and the investigation being of exploratory nature, the sample size of 48 should be sufficient to represent the varied veiws within the cohort. There are students in the study that represent all grade ranges and therefore should provide a representative picture of the entire cohort. To ensure free participation the focus group will be conducted after the marks are confirmed.

The novel method of collecting data prior and during the focus groups to encourage discussion suits the online video conferencing format.

Only 40 students $(31.49 \%)$ used paper logbooks as instructed by their Supervisors. Only prelimnary quantitative and qualitative online logbook data and some paper logbook data is presented in this paper. Detailed paper logbook data and complete online logbook data along further analysis will be published in a Journal in future.

\section{PURPOSE AND POTENTIAL HYPOTHESIS}

By studying student's motivation in using the two types of logbooks and investigating the ways in which they use the logbooks, it will be possible to assess the impact, if any, of either type of logbook on student's independent learning skills and difference in student behavior when using two different logbooks. This will help in developing support and training material for staff and students in order to promote independent learning through the use of logbooks.

Although at this stage the study is mainly exploratory, we expect some of the themes below to present themselves. We will collect data to test the listed hypothesis. The list below is not exhaustive, as the exploratory work will result in more potential hypotheses that we will need to test. Here are some of the potential hypotheses that we may be able to test in this study:

a. Auto locking online logbooks encourages students to record work regularly. We will look at how much and how frequent the entries are in the six online logbooks and /or hardbound paper logbook to prove/disprove this hypothesis. b. Online logbooks are as good as paper logbook when it comes to recording different types of entries. We will look for what is recorded in the two types of logbooks and compare the same to prove / disprove this hypothesis.

c. Irrespective of the type of logbook used, as students record more they begin to perform better reflections. We will look for some of the linguistic tools used that highlight reflections within the logbooks using work developed in $[3,8$ and 9] and others to prove/disprove this hypothesis.

\section{FINDINGS AND IMPLICATIONS}

So far only some of the data from a small sample of online logbooks have been extracted and analyzed. This was done to demonstrate the potential and the nature of results that this study will present when the work is completed. To this end, a randomly selected subset of students, one each from $40 \%$ of the Supervisors $(\mathrm{N}=20)$, was selected. This meant that data from 8 randomly selected student logbooks was extracted and analyzed so far. The students were not chosen on the basis of the type of logbook used. There is therefore an uneven mix of students in terms of the type of logbook used. Note that those who used paper logbooks also had to record at least the meeting minutes in their online logbooks.

The data represents first 12 weeks of student's work. The project runs for a total of 27 weeks. It is therefore expected that this analysis will be limited and not show the overall picture. No overall conclusions can yet be drawn from the analysis presented here. This will only be possible after the study and analysis is completed. All documents have now been submitted and projects marks but the focus group preparation is underway. These results will be published in a relevant journal.

Since the students used Google Docs for their e-logbooks, it was possible to check the date students actually updated their logbooks. Over the first 12 week period, there were a total of 66 attempts made by 8 students to create 106 unique entries, in real time or retrospectively. A total of 9275 words were typed in, resulting in 11 words per entry per student. A total of 41 images, 15 diagrams and or equations and 11 references and or links were used within the logbooks.

Over the 12-week period, on average some 2.5 entries per student $(18.8 \%)$ were done in real time, i.e. recorded as they worked. Over the same period an average of 4.75 entries $(35.8 \%)$ were more than 1 week late and 6 entries $(45.4 \%)$ were between 1 day and 1 week late. Therefore majority of the entries were recorded within 1 week from when they actually took place. However, a significant number of entries were written retrospectively $(81.2 \%)$. It is expected that this trend may continue as more logbooks are analyzed. A similar analysis of paper logbook is not possible so this aspect will be explored in the focus group.

Those who made more real time entries, tended to continue doing so, while others (majority) completed the logbooks in a retrospective manner, mostly within a week from the reported time of event being recorded. Analysis of remaining logbooks will show the trends and changes in the student behaviour when it comes to making timely log entries from one logbook to another over the entire project period. 
The students used their e-logbooks for recording the following types of entries, below is a select sample of the qualitative data we will be able to analyse:

a. Meeting minutes: "This week I have had a meeting with my project tutor to discuss the ideas that I have been considering over the previous week. We also agreed that I was to go away and create an outline for my preliminary report."

b. Links and references to useful resources: "Below are a list of resources I found: http://wwwijric.org/volumes/Vol4/7Vol4.pdf", the student lists 4 other papers and article and summarises them.

c. Summarizing articles read: "This article compared textual passwords with graphical passwords. I shown (sic) the advantages and disadvantages of both. This article did not implete (sic) an idea but was fantastic with the research."

d. Planning work for near future: "Need to set hypothesis to ask questions from. Need to test my authentication process by using and creating hardware/software to do it."

e. Problems faced: "The two issues I need to sort out is the Login page and the sequence creater (sic). Once the user picks their sequence it needs to be dispalyed (sic) in order. A macro code can solve this issues but getting it to work properly may be an issue. Another issue is the system can be alternated by the user i.e. formulas can be changed."

f. Reflections: "I need a lot of research to think of what sort of idea I can think of."

g. Relevant images scanned into the document: There were several examples of hand-drawn diagrams and images taken by students of their prototype, tests results, etc.

h. Relevant equations and diagrams: Several equations and diagrams were present in the logbooks and it seemed that these were created within Google docs.

i. Paper logbook scans: Not included here, but students scanned in to their online logbook, their doodles and diagrams which they chose to hand draw.

j. Plans and Gantt chart: Again these were presented in the initial logbooks of some students as tables, images etc. Shortterm plan updates were also present in logbooks.

A point that one can make from the above limited data is that paper logbooks are not very convenient to store URLs to visit resources, whereas the e-logbooks, being online resources, enable web links to work seamlessly. Equally, online logbooks may pose a barrier to some who are used to doodle by hand and pen as making diagrams online is not easy for everyone. We found that several students just scanned in the diagrams to address this. Many other students used the inbuilt or additional tools to create equations and make the diagrams or import images in their e-logbook.

An interesting observation from the data was that the Supervisors of two students commented on two of their elogbook entries. The Supervisors concerned provided feedback on student's previous work and gave guidance on future steps using the comment feature in Google docs. Where students received this type of support they increasingly made more realtime entries and less retrospective ones. This does not mean that for other students the feedback or guidance was missing or that other students did not make more real time entries. It only means that online logbooks can enable this kind of interaction and this may encourage students to update more often. In the process they can engage with feedback from their Supervisors. Paper logbooks are not shared in this way and may not enable this type of exchange. The paper logbooks too were used for reflections, summary of articles, planning, recording problem faced, hand drawing and doodling of ideas and concepts and pasting text from various sources after printing on paper to stick to the logbooks.

\section{REFERENCES}

[1] A. P. Maddocks, J. G. Dickens, A. R. Crawford, "Encouraging lifelong learning by means of a Web-based personal and professional development tool", ICEE 2002, UMIST, Manchester, 18-22 Aug, 8pp.

[2] H. McAlpine, B. J. Hicks, G. Huet and S. J. Culley, "An investigation into the use and content of the engineer's logbook", Design Studies vol 27 No. 4, July 2006, pp. 481-504

[3] P. J. Wild, C. McMahon, M. Darlington, S. Liu, \& S. Culley, “A diary study of information needs and document usage in the engineering domain", Design Studies, vol. 31 No.1, 2010, pp. 46-73.

[4] A. T. Kavelaara, E.Bloow, R. Claus, K. Fouts \& S. Tuvi, "Electronic logbook for space system integration \& test", IEEE Trans. on Aerospace and Electronic systems vol. 45, No. 1, January 2009, pp. 167-178,

[5] M. Malik, R. Khusainov, S. Zhou, \& V. Adamos, "A two year case study: Technology assisted project supervision (TAPaS)" Engineering education,vol. 4 No.2, 2009 pp. 76-83.

[6] P. E. Lonergan, J. Mulsow, W. A. Tanner, O. Traynor, \& S. Tierney, "Analysing the operative experience of basic surgical trainees in Ireland using a web-based logbook", BMC medical education, vol. 11, No.1, 2011, pp.70-78

[7] G. D. Denton, C. DeMott, L. N. Pangaro, \& P. A. Hemmer, "Narrative review: Use of student-generated logbooks in undergraduate medical education", Teaching and learning in medicine, vol. 18, No. 2, Jun 2010 , pp. 153-164.

[8] G. Campbell-Evans, \& C. Maloney, "An analysis framework for reflective writing", Australian Journal of Teacher Education, vol. 23 No.1, 1998, pp. 29-38.

[9] C. Reidsema \& P. Mort, "Assessing reflective writing: Analysis of reflective writing in an engineering design course" In Journal of Academic Language and Learning vol. 3, No. 2, 2009, pp. A-117- A129.

[10] J. Black, "The allocation and assessment of final year project work in the engineering degree course at the University of Bath", European Journal of Engineering Education, vol. 1, No. 1, 1975, pp. 56-68. 